\title{
ON THE DISTRIBUTION OF CHARACTERISTIC PARAMETERS OF WORDS II*
}

\author{
Arturo CARpi ${ }^{1}$ And Aldo De LuCA ${ }^{2}$
}

\begin{abstract}
The characteristic parameters $K_{w}$ and $R_{w}$ of a word $w$ over a finite alphabet are defined as follows: $K_{w}$ is the minimal natural number such that $w$ has no repeated suffix of length $K_{w}$ and $R_{w}$ is the minimal natural number such that $w$ has no right special factor of length $R_{w}$. In a previous paper, published on this journal, we have studied the distributions of these parameters, as well as the distribution of the maximal length of a repetition, among the words of each length on a given alphabet. In this paper we give the exact values of these distributions in a special case. However, these values give upper bounds to the distributions in the general case. Moreover, we study the most frequent and the average values of the characteristic parameters and of the maximal length of a repetition over the set of all words of length $n$.
\end{abstract}

Mathematics Subject Classification. 68R15, 68R05.

\section{INTRODUCTION}

In a recent paper [4], which hereafter will be also referred to as $\mathrm{CP}$, we have studied some properties of the distributions of two basic parameters which can be associated with any finite word $w$ on a given alphabet $A$. These parameters, called characteristic parameters and denoted by $K_{w}$ and $R_{w}$, are defined as follows: $K_{w}$ is the length of the shortest unrepeated suffix of $w$ and $R_{w}$ is the minimal natural

Keywords and phrases: Special factor, characteristic parameter, repeated factor.

* The work for this paper has been supported by the Italian Ministry of Education under Project COFIN 2001 - Linguaggi Formali e Automi: teoria ed applicazioni.

1 Dipartimento di Matematica e Informatica, Università di Perugia, via Vanvitelli 1, 06123 Perugia, Italy; e-mail: carpi@dipmat.unipg.it

2 Dipartimento di Matematica dell'Università di Roma "La Sapienza", piazzale Aldo Moro 2, 00185 Roma, Italy and Centro Interdisciplinare "B. Segre", Accademia dei Lincei, via della Lungara 10, 00100 Roma, Italy; e-mail: deluca@mat.uniroma1.it 
number such that $w$ has no right special factor of length $R_{w}$. We recall that a factor $u$ of a word $w$ is (right) special if there exist two distinct letters $a$ and $b$ such that $u a$ and $u b$ are both factors of $w$.

As shown in a series of papers [1-5] characteristic parameters give a great amount of information about the structure of a word. For instance, the maximal length $G_{w}$ of a repeated factor of a non-empty word $w$ is given by

$$
G_{w}=\max \left\{R_{w}, K_{w}\right\}-1
$$

In $\mathrm{CP}$ we studied how the values of the characteristic parameters, as well as of some other related quantities, are distributed among the words of each length. More precisely, if $A$ is a fixed $d$-letter alphabet, for any pair of natural numbers $i$ and $n$, we denote by $D_{R}(i, n), D_{K}(i, n)$, and $D_{G}(i, n)$ the number of words $w$ of length $n$ on the alphabet $A$ such that, respectively, $R_{w}, K_{w}$, and $G_{w}$ is equal to $i$. In the case of a binary alphabet, the values of $D_{R}(i, n) / 2, D_{K}(i, n) / 2$, and $D_{G}(i, n) / 2$ for small values of $i$ and $n$ are reported in Tables 1-3 of CP. The following basic relation between $D_{R}$ and $D_{G}$ holds: for all $i, n>0$ one has

$$
D_{R}(i, n+1)=D_{R}(i, n)+(d-1) D_{G}(i-1, n)
$$

Moreover, for $i, n>0$ one has

$$
D_{G}(i-1, n) \leq D_{R}(i, n)+D_{K}(i, n),
$$

where equality holds if and only if $i>n / 2$. We also showed that when $i$ is fixed and $n$ grows, $D_{R}(i, n)$ and $D_{K}(i, n)$ are non-decreasing. This is not true for $D_{G}(i, n)$, because one has $D_{G}(i, n) \neq 0$ if and only if $i<n \leq i+d^{i+1}$.

In CP we studied the "diagonal behaviour" of $D_{R}, D_{K}$, and $D_{G}$, i.e., the behaviour of $D_{R}(i, n), D_{K}(i, n)$, and $D_{G}(i, n)$ when variables $i$ and $n$ are simultaneously increased by 1 . We showed that, for any $i, n \geq 0$,

$$
D_{K}(i, n) \leq D_{K}(i+1, n+1)
$$

where equality holds if and only if $i>n / 2$. In other terms, for any fixed $m \geq 0$, the values of $D_{K}$ on the points of a diagonal line $(t, m+t)_{t \geq 0}$ are initially increasing and ultimately constant. A similar property holds for functions $D_{G}^{*}$ and $D_{K}^{*}$ where for all $i, n \geq 0, D_{G}^{*}(i, n)$ and $D_{K}^{*}(i, n)$ denote respectively the number of the words of length $n$ such that $G_{w} \geq i$ and $K_{w} \geq i$. Moreover, for $t, m \geq 0$, one has

$$
D_{R}(t, m+t) \leq D_{R}(m, 2 m)
$$

where the " $="$ sign holds if and only if $t \geq m$. Similarly, for $m>0$ and $t \geq 0$ one has

$$
D_{G}(t, m+t) \leq D_{G}(m, 2 m),
$$


where the " $=$ " sign holds if and only if $t \geq m-1$. When $i \geq n / 2$ some noteworthy relations hold. In particular, one has for $i \geq n / 2>0$

$$
D_{R}(i, n)=(d-1) d^{n-i} \sum_{t=1}^{n-i} d^{-t} D_{K}(t, 2 t-1)
$$

and, for $i \geq\lfloor n / 2\rfloor \geq 1$

$$
D_{R}(i, n)=(d-1) D_{G}^{*}(i-1, n-1) .
$$

In this paper we continue the analysis of the distributions of characteristic parameters of words. In Section 2 we obtain explicit arithmetic expressions, involving the Möbius function, for $D_{R}(i, n), D_{K}(i, n), D_{G}(i, n), D_{K}^{*}(i, n)$, and $D_{G}^{*}(i, n)$, at least when $i>n / 2$. In view of the "diagonal behaviour", these expressions give upper bounds to the values of the preceding maps, in the general case.

Another result concerns the counting of repetitions. By repetition of length $m$ in a word $w$ we mean any unordered pair of distinct occurrences of the same factor of length $m$ in $w$. We show that the total number of repetitions of length $m$ in all the words of length $n$ on a $d$-letter alphabet is given by

$$
d^{n-m}\left(\begin{array}{c}
n-m+1 \\
2
\end{array}\right)
$$

This and other related results are of interest for applications, since repetitions play an essential role in algorithms for text compression and sequence assembly $[5,8,10]$.

In the last two sections we study the behaviour of $D_{R}(i, n), D_{K}(i, n)$, and $D_{G}(i, n)$ when the length $n$ is fixed and $i$ varies. In Section 3 we are mainly interested in the average values $\langle R\rangle_{n},\langle K\rangle_{n},\langle G\rangle_{n}$ of $R_{w}, K_{w}$, and $G_{w}$ on the words $w$ of length $n$ on a $d$-letter alphabet, with $d \geq 2$. We study the most frequent values of the characteristic parameters and of the maximal length of a repetition in the set of words of length $n$ and use these results for evaluating the average values. We show that $\langle G\rangle_{n}$ and $\langle K\rangle_{n}$ are upperbounded, respectively, by $\left\lceil 2 \log _{d} n\right\rceil-1 / 2$ and $\left\lceil\log _{d} n\right\rceil+2$ while $\langle R\rangle_{n}$ is lowerbounded by $\left\lfloor\log _{d}(n-1)\right\rfloor$. Moreover,

$$
\lim _{n \rightarrow \infty} \frac{\langle K\rangle_{n}}{\log _{d} n}=\lim _{n \rightarrow \infty}\left(\langle R\rangle_{n}-\langle G\rangle_{n}\right)=1
$$

and

$$
\lim _{n \rightarrow \infty}\left(\langle G\rangle_{n}-\langle G\rangle_{n-1}\right)=\lim _{n \rightarrow \infty}\left(\langle R\rangle_{n}-\langle R\rangle_{n-1}\right)=\lim _{n \rightarrow \infty}\left(\langle K\rangle_{n}-\langle K\rangle_{n-1}\right)=0 .
$$

We also obtain upper bounds to the number of symmetric words ( $c f$. Sect. 3 of $\mathrm{CP}$ ) of length smaller than $n$ and to the number of semiperiodic words [2] of length $n$. Moreover, we prove that the fraction of the words of length $n$ which are periodic-like [3] is exactly given by $\langle K\rangle_{n}-\langle K\rangle_{n-1}$. 
In Section 4 we show that the points of maximum of $D_{G}(i, n)$, viewed as a function of $i$ with $n$ fixed but sufficiently large, lie between $\left\lfloor\log _{d} n\right\rfloor-1$ and $\left\lceil 2 \log _{d} n+\right.$ $\left.\log _{d} \log _{d} n\right\rceil-1$. Similarly, the points of maximum of $D_{R}(i, n)$ and $D_{K}(i, n)$ lie, respectively, between $\left\lfloor\log _{d} n-\log _{d} \log _{d} n\right\rfloor-4$ and $\left\lceil 2 \log _{d} n+\log _{d} \log _{d} n\right\rceil$ and between 0 and $\left\lceil\log _{d} n+\log _{d} \log _{d} n\right\rceil+2$.

\section{Preliminaries}

Let $A$ be a non-empty set, or alphabet, of cardinality $d>0$. We denote by $A^{*}$ the set of all finite sequences of elements of $A$, including the empty sequence, denoted by $\epsilon$. The elements of $A$ are usually called letters and those of $A^{*}$ words. The word $\epsilon$ is called empty word. We set $A^{+}=A^{*} \backslash\{\epsilon\}$. A word $w \in A^{+}$can be written uniquely as a sequence of letters as

$$
w=a_{1} a_{2} \cdots a_{n}
$$

with $a_{i} \in A, 1 \leq i \leq n, n>0$. The integer $n$ is called the length of $w$ and denoted by $|w|$. By definition, the length of $\epsilon$ is equal to 0 . For any $n \geq 0$ we set $A^{n}=\left\{w \in A^{*}|| w \mid=n\right\}$.

A word $w$ is called primitive if it cannot be written as $w=u^{r}$ with $u \neq \epsilon$ and $r>1$. Two words $u, v \in A^{*}$ are conjugate if there exist words $r, s \in A^{*}$ such that $u=r s$ and $v=s r$. As is well known (see [9]), conjugacy is an equivalence relation in $A^{*}$. Moreover, any conjugate of a primitive word is primitive. A conjugacy class of a primitive word will be called primitive.

Let $w \in A^{*}$. The word $u \in A^{*}$ is a factor (or subword) of $w$ if there exist words $\lambda, \mu$ such that $w=\lambda u \mu$. A factor $u$ of $w$ is called proper if $u \neq w$. If $w=u \mu$, for some word $\mu$ (resp. $w=\lambda u$, for some word $\lambda$ ), then $u$ is called a prefix (resp. suffix) of $w$. For any word $w$, we denote respectively by $\operatorname{Fact}(w), \operatorname{Pref}(w)$, and $\operatorname{Suff}(w)$ the sets of its factors, prefixes, and suffixes.

Let $u \in \operatorname{Fact}(w)$. Any pair $(\lambda, \mu) \in A^{*} \times A^{*}$ such that $w=\lambda u \mu$ is called an occurrence of $u$ in $w$. If $\lambda=\epsilon$ (resp. $\mu=\epsilon$ ), then the occurrence of $u$ is called initial (resp. terminal). An occurrence is called internal if it is neither initial nor terminal. A factor $u$ of $w$ is repeated if it has at least two distinct occurrences in $w$, otherwise it is called unrepeated.

A word $s$ is called a right (resp. left) special factor of $w$ if there exist two letters $x, y \in A, x \neq y$, such that $s x, s y \in \operatorname{Fact}(w)(\operatorname{resp} . x s, y s \in \operatorname{Fact}(w))$.

With each word $w$ one can associate the word $k_{w}$ (resp. $h_{w}$ ) defined as the shortest suffix (resp. prefix) of $w$ which is an unrepeated factor of $w$.

In the following, for any non-empty word $w$, we shall denote by $k_{w}^{\prime}\left(\right.$ resp. $\left.h_{w}^{\prime}\right)$ the longest repeated suffix (resp. prefix) of $w$. One has, trivially, $k_{w}=x k_{w}^{\prime}$ and $h_{w}=h_{w}^{\prime} y$ with $x, y \in A$.

For any word $w$, we shall consider the parameters $K_{w}=\left|k_{w}\right|$ and $H_{w}=\left|h_{w}\right|$. Moreover, we shall denote by $R_{w}$ the minimal natural number such that there is no right special factor of $w$ of length $R_{w}$ and by $L_{w}$ the minimal natural number such that there is no left special factor of $w$ of length $L_{w}$. 
For any $w \in A^{+}$we set $B_{w}=\left\{a \in A \mid k_{w}^{\prime} a \in \operatorname{Fact}(w)\right\}$. Thus, $B_{w}$ is the set of letters of $A$ extending on the right $k_{w}^{\prime}$ in $w$. Moreover, we set $B_{\epsilon}=A$. In Section 2 of CP we proved that for all $w \in A^{*}$ and any $x \in B_{w}$ one has

$$
K_{w x}=K_{w}+1 \quad \text { and } \quad R_{w x}=R_{w}
$$

Let $w=a_{1} \cdots a_{n}, a_{r} \in A, 1 \leq r \leq n$. A repetition of length $q>0$ in $w$ is any pair $(i, j), 1 \leq i<j \leq n-q+1$, such that

$$
a_{i} a_{i+1} \cdots a_{i+q-1}=a_{j} a_{j+1} \cdots a_{j+q-1} .
$$

Moreover, a repetition of length 0 is any pair $(i, j)$ with $1 \leq i<j \leq n+1$.

The maximal length of a repetition in $w$, that is the maximal length of a repeated factor of $w$, is denoted by $G_{w}$. As proved in Section 3 of $\mathrm{CP}$, for all $w \in A^{+}$ one has

$$
G_{w}+1 \leq|w| \leq G_{w}+d^{R_{w}}
$$

In particular, if $d>1$ one derives

$$
G_{w} \geq\left\lfloor\log _{d}|w|\right\rfloor-1
$$

The following lemmas will be useful in the sequel:

Lemma 1.1. Let $d>1$. For any $w \in A^{+}$one has

$$
G_{w}+R_{w} \geq 2\left\lfloor\log _{d}|w|\right\rfloor-1 \text {. }
$$

Proof. Let $w \in A^{n}$. By equation (10), $G_{w}+R_{w} \geq G_{w}+\log _{d}\left(n-G_{w}\right)$. Since the second derivative of the function $x+\log _{d}(n-x)$ is negative, the minimal value of this function in the interval $\left[\left\lfloor\log _{d} n\right\rfloor-1, n-1\right]$ is equal to

$$
\min \left\{\left\lfloor\log _{d} n\right\rfloor-1+\log _{d}\left(n-\left\lfloor\log _{d} n\right\rfloor+1\right), n-1\right\}
$$

By equations (10) and (11), $\left\lfloor\log _{d} n\right\rfloor-1 \leq G_{w} \leq n-1$ so that one derives

$$
G_{w}+R_{w} \geq \min \left\{\left\lfloor\log _{d} n\right\rfloor-1+\log _{d}\left(n-\left\lfloor\log _{d} n\right\rfloor+1\right), n-1\right\}
$$

Since for $d \geq 2$ one has $n \geq d\left\lfloor\log _{d} n\right\rfloor$, one gets

$$
n-1 \geq 2\left\lfloor\log _{d} n\right\rfloor-1
$$

and

$$
n-\left\lfloor\log _{d} n\right\rfloor+1 \geq n-\frac{n}{d}+1>\frac{n}{d},
$$


so that

$$
\left\lfloor\log _{d} n\right\rfloor-1+\log _{d}\left(n-\left\lfloor\log _{d} n\right\rfloor+1\right)>\left\lfloor\log _{d} n\right\rfloor-1+\log _{d} \frac{n}{d} \geq 2\left\lfloor\log _{d} n\right\rfloor-2 .
$$

By equations $(12,13)$, and $(14)$ one obtains $G_{w}+R_{w}>2\left\lfloor\log _{d} n\right\rfloor-2$, from which the conclusion follows.

We observe that the lower bound in the preceding lemma is effectively reached. In fact, if $w$ is a de Bruijn word of order $m$ ( $c f$. Sect. 3 of CP) one has $R_{w}=m$, $G_{w}=m-1$, and $m=\left\lfloor\log _{d}|w|\right\rfloor$.

Lemma 1.2. Let $d>1$. For any $w \in A^{+}$such that $R_{w}<\left\lfloor\log _{d}|w|\right\rfloor$ one has $R_{w}+K_{w} \geq 2\left\lfloor\log _{d}|w|\right\rfloor$.

Proof. By Lemma 1.1 one has $G_{w} \geq 2\left\lfloor\log _{d}|w|\right\rfloor-1-R_{w} \geq\left\lfloor\log _{d}|w|\right\rfloor>R_{w}$. This implies $K_{w}=G_{w}+1$ so that $R_{w}+K_{w} \geq 2\left\lfloor\log _{d}|w|\right\rfloor$.

Let us denote by $P_{w}(q)$ the number of all repetitions of length $q$ in $w$. For instance, in the case of the word $w=a a b a a b a b b a b$, as one easily verifies, one has $P_{w}(1)=25, P_{w}(2)=10, P_{w}(3)=3, P_{w}(4)=1$, and $P_{w}(5)=0$.

Lemma 1.3. For any $w \in A^{+}$and $q \geq 0$, one has

$$
P_{w}(q) \geq G_{w}-q+1
$$

Proof. The result is trivially true if $q=0$ or $q>G_{w}$. Thus suppose $0<q \leq G_{w}$. Let us write $w$ as $w=a_{1} \cdots a_{n}$ with $a_{r} \in A, 1 \leq r \leq n$. Since $G_{w}$ is the maximal length of a repeated factor of $w$ there exist integers $i$ and $j$ such that $1 \leq i<j \leq n-G_{w}+1$ and

$$
a_{i} a_{i+1} \cdots a_{i+G_{w}-1}=a_{j} a_{j+1} \cdots a_{j+G_{w}-1} .
$$

Thus, the $G_{w}-q+1$ pairs

$$
(i, j),(i+1, j+1), \ldots,\left(i+G_{w}-q, j+G_{w}-q\right)
$$

are repetitions of length $q$ of $w$. Hence, $P_{w}(q) \geq G_{w}-q+1$.

\section{Exact COMputations}

In the sequel we shall assume that the alphabet $A$ contains at least two letter, i.e., $d>1$.

In this section we give explicit arithmetic expressions for $D_{R}(i, n), D_{K}(i, n)$, $D_{G}(i, n), D_{K}^{*}(i, n)$, and $D_{G}^{*}(i, n)$, involving the Möbius function, at least when $i>n / 2$. In view of the diagonal behaviour of these functions, these expressions give upper bounds to the values of the preceding maps, when $i \leq n / 2$. 
Let $w=a_{1} a_{2} \cdots a_{n}$ be a word, $a_{i} \in A, i=1, \ldots, n$. We recall (cf. [9]) that a positive integer $p \leq n$ is called a period of $w$ if for all $i, j \in[1, n]$ such that $i \equiv j$ $(\bmod p)$, one has $a_{i}=a_{j}$. For any word $w$, we denote by $\pi_{w}$ its minimal period. A word $w$ is called periodic if $|w| \geq 2 \pi_{w}$.

The notion of period is also related to the notion of border of a word. A word $u$ is called a border of $w$ if it is both a proper prefix and a proper suffix of $w$. The longest border of the word $w$ will be called the maximal border of $w$. It is well known (cf. [9]) that the maximal border of a word $w$ has length $|w|-\pi_{w}$.

Let $\psi: \mathbb{N}_{+} \rightarrow \mathbb{N}_{+}$be the function counting, for any positive integer $n$ the number of primitive words of length $n$ on the alphabet $A$. As is well known [9], for any $n, \psi(n)$ is given by

$$
\psi(n)=\sum_{m \mid n} \mu(m) d^{\frac{n}{m}},
$$

where $\mu$ is the Möbius function (see, for instance [7]).

Lemma 2.1. Let $n$ and $p$ be positive integers such that $n \geq 2 p-2$. The number of words of length $n$ having minimal period $p$ is given by $\psi(p)$.

Proof. Let $u$ be a primitive word of length $p$ and prolong $u$ on the right in a word $w$ of length $n$ having period $p$. Let us show that $p$ is the minimal period of $w$. Indeed, suppose that $w$ has a minimal period $q<p$. Since $n \geq 2 p-2 \geq p+q-1$, by the theorem of Fine and Wilf [6], w has also the period $\operatorname{gcd}(p, q)$ which has to be equal to $q$, since $q$ is the minimal period of $w$. Thus, $p=r q$ with $r>1$. Since $u$ has the period $q$, it follows that $u$ is not primitive, which is a contradiction. Conversely, let $w$ be a word of length $n$ having the minimal period $p$. Then the prefix $u$ of length $p$ of $w$ has to be primitive as, otherwise, the minimal period of $w$ would be less than $p$.

In conclusion, if $n \geq 2 p-2$, the number of words of length $n$ having minimal period $p$ coincides with the number of primitive words of length $p$, i.e., $\psi(p)$.

Lemma 2.2. Let $m$ be a positive integer and $w$ a word. One has $K_{w} \geq m$ if and only if there exists $u \in \operatorname{Suff}(w)$ such that $|u|=\pi_{u}+m-1$.

Proof. Let us suppose that $K_{w} \geq m$. Thus $w$ has a repeated suffix $v$ of length $m-1$. Let $u$ be the shortest suffix of $w$ with two occurrences of $v$. This implies that $v$ is a border of $u$ with no internal occurrence in $u$. Moreover, $v$ is the longest border of $u$, otherwise $v$ would have an internal occurrence in $u$. Hence, the minimal period of $u$ is given by $\pi_{u}=|u|-|v|=|u|-m+1$.

Conversely, suppose that there exists $u \in \operatorname{Suff}(w)$ such that $|u|=\pi_{u}+m-1$. Then, $u$ has a maximal border $v$ of length $|v|=|u|-\pi_{u}=m-1$. The word $v$ is a repeated suffix of $w$ so that $K_{w} \geq|v|+1=m$.

Lemma 2.3. Let $w$ be a word and $m>|w| / 2$. Then there is at most one suffix $u$ of $w$ such that $|u|=\pi_{u}+m-1$. 
Proof. Suppose that $u, u^{\prime} \in \operatorname{Suff}(w)$ are such that $|u|=\pi_{u}+m-1,\left|u^{\prime}\right|=\pi_{u^{\prime}}+$ $m-1$, and $\left|u^{\prime}\right| \leq|u|$. Since $|u| \leq|w| \leq 2 m-1$, it follows that $\pi_{u} \leq m$ so that

$$
\left|u^{\prime}\right|=\pi_{u^{\prime}}+m-1 \geq \pi_{u^{\prime}}+\pi_{u}-1 .
$$

Since $u^{\prime}$ is a suffix of $u, u^{\prime}$ has also the period $\pi_{u}$. By the theorem of Fine and Wilf [6], it follows that $u^{\prime}$ has the period $\operatorname{gcd}\left(\pi_{u}, \pi_{u^{\prime}}\right)$. Thus, since $\pi_{u^{\prime}}$ is the minimal period of $u^{\prime}$ one has $\pi_{u^{\prime}}=\operatorname{gcd}\left(\pi_{u}, \pi_{u^{\prime}}\right)$, so that $\pi_{u}$ is a multiple of $\pi_{u^{\prime}}$. Since $\pi_{u} \leq\left|u^{\prime}\right|$ and $\pi_{u}$ is a multiple of $\pi_{u^{\prime}}$ it follows that $\pi_{u^{\prime}}$ is a period of $u$. Consequently, $\pi_{u^{\prime}}=\pi_{u}$ and $|u|=\left|u^{\prime}\right|$ which implies $u=u^{\prime}$.

We recall that the map $D_{K}^{*}$ is defined for all $i, n \geq 0$ by

$$
D_{K}^{*}(i, n)=\operatorname{Card}\left(\left\{w \in A^{n} \mid K_{w} \geq i\right\}\right)=\sum_{m \geq i} D_{K}(m, n) .
$$

By equation (3) one easily derives ( $c f$. Sect. 5 of CP) that for all $i, n \geq 0$,

$$
D_{K}^{*}(i, n) \leq D_{K}^{*}(i+1, n+1),
$$

where equality holds if and only if $i>n / 2$.

Proposition 2.4. Let $m$ and $n$ be integers with $0 \leq m \leq n$. One has

$$
D_{K}^{*}(m, n) \leq \sum_{i=1}^{n-m+1} d^{n-m-i+1} \psi(i)
$$

where equality holds if and only if $m>n / 2$. In particular, for $0 \leq m \leq n$ one has

$$
D_{K}^{*}(m, n) \leq(n-m+1) d^{n-m+1} .
$$

Proof. First, we suppose that $m>n / 2$. Then, for any $i=1, \ldots, n-m+1$, one has $i+m-1 \geq 2 i-1$ so that, by Lemma 2.1 there are exactly $\psi(i)$ words of minimal period $i$ and length $i+m-1$. These words can be prolonged on the left into $d^{n-m-i+1} \psi(i)$ words of length $n$ satisfying the condition in Lemma 2.2. Since $m>n / 2$, by using Lemma 2.3, one derives that, starting from distinct values of the period $i$, distinct words are obtained. We conclude that the total number of words $w$ of length $n$ such that $K_{w} \geq m$, i.e., $D_{K}^{*}(m, n)$ is given by $\sum_{i=1}^{n-m+1} d^{n-m-i+1} \psi(i)$.

If, on the contrary, $m \leq n / 2$, then by equation (15) and the first part of the proof one has

$$
D_{K}^{*}(m, n)<D_{K}^{*}(m+n+1,2 n+1)=\sum_{i=1}^{n-m+1} d^{n-m-i+1} \psi(i) .
$$


To conclude the proof, we observe that for any $i \geq 1$, trivially $\psi(i) \leq d^{i}$, so that

$$
\sum_{i=1}^{n-m+1} d^{n-m-i+1} \psi(i) \leq \sum_{i=1}^{n-m+1} d^{n-m+1}=(n-m+1) d^{n-m+1}
$$

In the sequel, we follow the convention that a sum $\sum_{i=t}^{s} a_{i}$ holds 0 if $t>s$.

Proposition 2.5. Let $m$ and $n$ be integers with $0 \leq m \leq n$. One has

$$
D_{K}(m, n) \leq \psi(n-m+1)+d^{n-m}(d-1) \sum_{i=1}^{n-m} d^{-i} \psi(i)
$$

where equality holds if and only if $m>n / 2$.

Proof. One can write

$$
D_{K}(m, n)=\sum_{i \geq m} D_{K}(i, n)-\sum_{i \geq m+1} D_{K}(i, n)=D_{K}^{*}(m, n)-D_{K}^{*}(m+1, n) .
$$

If $m>n / 2$, by Proposition 2.4 one has

$$
\begin{aligned}
D_{K}(m, n) & =\sum_{i=1}^{n-m+1} d^{n-m-i+1} \psi(i)-\sum_{i=1}^{n-m} d^{n-m-i} \psi(i) \\
& =\psi(n-m+1)+d^{n-m}(d-1) \sum_{i=1}^{n-m} d^{-i} \psi(i) .
\end{aligned}
$$

If, on the contrary, $m \leq n / 2$, then, by an iterated application of equation (3), one derives

$$
D_{K}(m, n)<D_{K}(m+n+1,2 n+1) .
$$

Since $m+n+1>(2 n+1) / 2$, by the previous argument it follows

$$
D_{K}(m, n)<\psi(n-m+1)+d^{n-m}(d-1) \sum_{i=1}^{n-m} d^{-i} \psi(i)
$$

which concludes the proof.

Let us introduce now the function $\omega$ defined for any $p \geq 0$ by

$$
\omega(p)=\sum_{t=1}^{p} d^{-t-1} \psi(t)(d+(d-1)(p-t))
$$


Proposition 2.6. Let $m, n$ be integers with $0 \leq m \leq n$. One has

$$
D_{R}(m, n) \leq(d-1) d^{n-m} \omega(n-m),
$$

where equality holds if and only if $m \geq n / 2$.

Proof. The statement is trivial for $n=0$. Let us then suppose $n>0$.

First we consider the case $m \geq n / 2$. By equation (6) one has

$$
D_{R}(m, n)=(d-1) d^{n-m} \sum_{t=1}^{n-m} d^{-t} D_{K}(t, 2 t-1) \text {. }
$$

For $1 \leq t \leq n-m$ one has $(2 t-1) / 2<t \leq 2 t-1$ so that, by Proposition 2.5

$$
D_{K}(t, 2 t-1)=\psi(t)+d^{t-1}(d-1) \sum_{i=1}^{t-1} d^{-i} \psi(i)
$$

Thus,

$$
D_{R}(m, n)=(d-1) d^{n-m}\left(\sum_{t=1}^{n-m} d^{-t} \psi(t)+\frac{d-1}{d} \sum_{t=1}^{n-m} \sum_{i=1}^{t-1} d^{-i} \psi(i)\right) .
$$

Since

$$
\sum_{t=1}^{n-m} \sum_{i=1}^{t-1} d^{-i} \psi(i)=\sum_{i=1}^{n-m}(n-m-i) \psi(i) d^{-i}
$$

equation (17) becomes

$$
\begin{aligned}
D_{R}(m, n) & =(d-1) d^{n-m} \sum_{t=1}^{n-m} d^{-t} \psi(t)\left(1+\frac{d-1}{d}(n-m-t)\right) \\
& =(d-1) d^{n-m} \omega(n-m) .
\end{aligned}
$$

In the general case, by equation (4) one derives $D_{R}(m, n) \leq D_{R}(n-m, 2(n-m))$ and, by the previous argument, $D_{R}(m, n) \leq(d-1) d^{n-m} \omega(n-m)$, where equality holds if and only if $m \geq n / 2$. This proves our assertion.

We recall that the map $D_{G}^{*}$ is defined for $i \geq 0$ and $n>0$ by

$$
D_{G}^{*}(i, n)=\operatorname{Card}\left(\left\{w \in A^{n} \mid G_{w} \geq i\right\}\right)=\sum_{m \geq i} D_{G}(m, n) .
$$

As proved in Section 5 of CP, for any $i \geq 0$ and $n>0$ one has

$$
D_{G}^{*}(i, n) \leq D_{G}^{*}(i+1, n+1)
$$

where equality holds if and only if $i \geq\lfloor n / 2\rfloor$. 
Proposition 2.7. Let $m, n$ be integers with $0 \leq m<n$. One has

$$
D_{G}^{*}(m, n) \leq d^{n-m} \omega(n-m)
$$

where equality holds if and only if $m \geq\lfloor n / 2\rfloor$.

Proof. Let us first suppose that $\lfloor n / 2\rfloor \leq m<n$. Since $m+1 \geq(n+1) / 2$, by Proposition 2.6 one has $D_{R}(m+1, n+1)=(d-1) d^{n-m} \omega(n-m)$. Moreover, by equation $(7), D_{R}(m+1, n+1)=(d-1) D_{G}^{*}(m, n)$. This implies $D_{G}^{*}(m, n)=$ $d^{n-m} \omega(n-m)$.

In the case $m<\lfloor n / 2\rfloor$, by equation (18), one derives $D_{G}^{*}(m, n)<D_{G}^{*}(m+$ $n, 2 n)$. Since $m+n \geq n$, one has $D_{G}^{*}(m+n, 2 n)=d^{n-m} \omega(n-m)$, and this proves the assertion.

The previous proposition gives an upper bound to the number of words having at least one repeated factor of length $m$. Observe that, since $\psi(t) \leq d^{t}, t \geq 1$, for any $p>0$, one has

$$
\omega(p)<\sum_{t=1}^{p} d^{-t} \psi(t)(1+p-t)<\sum_{t=1}^{p}(1+p-t)=\left(\begin{array}{c}
p+1 \\
2
\end{array}\right) .
$$

Thus, a less sharp but simpler upper bound to $D_{G}^{*}(m, n)$ is given by the following corollary. A similar upper bound was proved recently in [10].

Corollary 2.8. For $0 \leq m<n$ the following holds:

$$
D_{G}^{*}(m, n)<d^{n-m}\left(\begin{array}{c}
n-m+1 \\
2
\end{array}\right) .
$$

An interpretation of this upper bound can be given in terms of the total number $P(m, n)$ of repetitions of length $m$ in all the words of length $n$, i.e.,

$$
P(m, n)=\sum_{w \in A^{n}} P_{w}(m)
$$

Proposition 2.9. Let $n$ and $m$ be integers such that $0 \leq m<n$. The following holds:

$$
P(m, n)=d^{n-m}\left(\begin{array}{c}
n-m+1 \\
2
\end{array}\right) .
$$

Proof. If $m=0$, then the result is trivial. Thus, we suppose $m>0$. For any pair of integers $i$ and $j$ such that $1 \leq i<j \leq n-m+1$, we count the words $w=a_{1} \cdots a_{n}$, $a_{r} \in A, 1 \leq r \leq n$, satisfying equation (9) with $q=m$. Let us prove that a word $w$ satisfying equation (9) is uniquely determined by the word $a_{1} \cdots a_{j-1} a_{j+m} \cdots a_{n}$. Indeed, by equation (9) one has $a_{j+p}=a_{i+p}, 0 \leq p \leq m-1$. One derives $a_{j}=a_{i}$. If we suppose of knowing all the letters up to $a_{j+p-1}, 0<p \leq m-1$, then $a_{j+p}=a_{i+p}$ and $a_{i+p}$ is already known since $i+p<j+p$. This proves that the 
number of the words $w$ satisfying equation (9) is given by $d^{n-m}$. Since there are $(n-m+1)(n-m) / 2$ pairs $(i, j)$ satisfying the condition $1 \leq i<j \leq n-m+1$ the result follows.

From Proposition 2.9, one obtains a different proof of Corollary 2.8 since, trivially, $D_{G}^{*}(m, n) \leq P(m, n)$.

Example 2.10. Let us consider a binary alphabet and let $m=5$ and $n=18$. In this case by means of a computer one obtains $D_{G}^{*}(5,18)=\sum_{i=5}^{18} D_{G}(i, 18)=$ 223250 (cf. Tab. 3 of CP). By Proposition 2.7 one has $D_{G}^{*}(5,18) \leq 2^{13} \omega(13)=$ $D_{G}^{*}(12,25)=363874<P(5,18)=745472$.

Proposition 2.11. Let $m$ and $n$ be integers with $0 \leq m \leq n$. One has

$$
\begin{aligned}
& D_{G}(m, n) \leq \psi(n-m) \\
& \quad+(d-1) d^{n-m-2} \sum_{t=1}^{n-m-1} d^{-t} \psi(t)(2+(d-1)(n-m-t+1)),
\end{aligned}
$$

where equality holds if and only if $m \geq\lfloor n / 2\rfloor$.

Proof. If $m \geq\lfloor n / 2\rfloor$, by Proposition 2.7 one has

$D_{G}(m, n)=D_{G}^{*}(m, n)-D_{G}^{*}(m+1, n)=d^{n-m} \omega(n-m)-d^{n-m-1} \omega(n-m+1)$.

In view of equation (16), by simple algebraic manipulations, one derives

$$
\begin{aligned}
& D_{G}(m, n)=\psi(n-m) \\
& +(d-1) d^{n-m-2} \sum_{t=1}^{n-m-1} d^{-t} \psi(t)(2+(d-1)(n-m-t+1)) .
\end{aligned}
$$

In the general case, by equation (5) one derives $D_{G}(m, n) \leq D_{G}(n-m-1,2(n-$ $m)-1)$, where equality holds if and only if $m \geq\lfloor n / 2\rfloor$. By the previous argument the result follows.

In conclusion of this section, we compute the exact value of $D_{K}(2, n), n \geq 0$, in the case of a binary alphabet. We denote by $\mathrm{Fib}_{n}$ the sequence of Fibonacci numbers, defined by

$$
\text { Fib }_{0}=0, \quad \text { Fib }_{1}=1, \quad \text { Fib }_{n+1}=\text { Fib }_{n}+\text { Fib }_{n-1}, n \geq 1 .
$$

Proposition 2.12. Let $d=2$. For all $n>1$ one has

$$
\frac{1}{2} D_{K}(2, n)=\mathrm{Fib}_{n-1}+n-2 .
$$


Proof. For $n=2$, the result is trivial since $D_{K}(2,2)=2$ and Fib $1=1$. Let us suppose $n>2$.

We have to count the number of words $w \in\{a, b\}^{n}$ having $K_{w}=2$. For symmetry reasons, we shall count only the words ending by the letter $a$.

First, we consider the case $k_{w}=b a$. Thus the letter $a$, but not $b a$, has to appear in the prefix of $w$ of length $n-2$. Any occurrence of $a$ in this prefix either is the prefix of $w$ of length 1 or is preceded by another $a$. Hence, the only possible words of this kind are $a^{j} b^{m} a$ with $j, m>0$ and $j+m+1=n$. Their number is $n-2$.

Now, we consider the case $k_{w}=a a$. We observe that one has $k_{w}=a a$ if and only if $w=b u$ or $w=a b u$ with $k_{u}=a a$. Indeed, since $|w|>2, w$ cannot begin by $a a$, so that either $w=b u$ or $w=a b u$. Moreover, $a a$ is an unrepeated suffix of $w$ if and only if it is an unrepeated suffix of $u$. Let us denote by $g(n)$ the number of words $w \in\{a, b\}^{n}$ such that $k_{w}=a a$. The previous argument shows that, for $n>2, g(n)=g(n-1)+g(n-2)$. Since $g(1)=0=\mathrm{Fib}_{0}$ and $g(2)=1=\mathrm{Fib}_{1}$, it follows that $g(n)=\mathrm{Fib}_{n-1}$.

Therefore, the total number of words $w$ of length $n$ ending by $a$ and having $K_{w}=2$ is given by $\mathrm{Fib}_{n-1}+n-2$.

From the previous proposition, in the case $d=2$ one easily derives that for $n \geq 4$ one has $D_{K}(2, n)=D_{K}(2, n-1)+D_{K}(2, n-2)-2(n-5)$. An interesting problem is to determine in the case $i>2$ similar recursive relations for $D_{K}(i, n)$.

\section{Average values}

In this section we shall be mainly interested in the average values $\langle R\rangle_{n},\langle K\rangle_{n}$, $\langle G\rangle_{n}$ of $R_{w}, K_{w}$, and $G_{w}$ on the words $w$ of length $n$ on the alphabet $A$. First we evaluate the most frequent values of the characteristic parameters and of the maximal length of a repetition in the set of words of length $n$. From this, we show that $\langle G\rangle_{n}$ and $\langle K\rangle_{n}$ are upperbounded, respectively, by $\left\lceil 2 \log _{d} n\right\rceil-1 / 2$ and $\left\lceil\log _{d} n\right\rceil+2$ while $\langle R\rangle_{n}$ is lowerbounded by $\left\lfloor\log _{d}(n-1)\right\rfloor$. Moreover, one has $\lim _{n \rightarrow \infty}\langle K\rangle_{n} / \log _{d} n=1, \lim _{n \rightarrow \infty}\left(\langle R\rangle_{n}-\langle G\rangle_{n}\right)=1$, and $\lim _{n \rightarrow \infty}\left(\langle G\rangle_{n}-\right.$ $\left.\langle G\rangle_{n-1}\right)=\lim _{n \rightarrow \infty}\left(\langle R\rangle_{n}-\langle R\rangle_{n-1}\right)=\lim _{n \rightarrow \infty}\left(\langle K\rangle_{n}-\langle K\rangle_{n-1}\right)=0$. We also obtain upper bounds to the number of symmetric words of length smaller than $n$ and to the number of semiperiodic words [2] of length $n$. Moreover, we show that the number of periodic-like words of length $n$ is equal to $d^{n}\left(\langle K\rangle_{n}-\langle K\rangle_{n-1}\right)$.

We start with the following proposition showing that the words of length $n$ having a repeated factor of length significantly larger than $2 \log _{d} n$ are a small fraction of all words of length $n$. This result was first proved in [10].

Proposition 3.1. Let $n>1$ and $r \geq 0$. The following holds:

$$
\operatorname{Card}\left(\left\{w \in A^{n} \mid G_{w} \geq\left\lceil 2 \log _{d} n\right\rceil+r\right\}\right)<\frac{1}{2} d^{n-r} .
$$

Proof. Let us set $m=\left\lceil 2 \log _{d} n\right\rceil+r$. If $m \geq n$, the result is trivially true, because the set $\left\{w \in A^{n} \mid G_{w} \geq m\right\}$ is empty. Let us then suppose $m<n$. Since $m>0$, 
we can write

$$
\left(\begin{array}{c}
n-m+1 \\
2
\end{array}\right) d^{n-m}<\frac{n^{2}}{2} d^{n-m}<\frac{1}{2} d^{n-r} .
$$

From Corollary 2.8 the result follows:

By the previous proposition one has that the number of words $w$ of length $n$ such that $G_{w}<\left\lceil 2 \log _{d} n\right\rceil+r$ is greater than $d^{n}\left(1-d^{-r} / 2\right)$. In particular, if one takes $r=\left\lfloor\log _{d} \log _{d} n\right\rfloor$, then by the preceding formula and equation (11) one derives that, for a sufficiently large $n$, the maximal length of repetitions in the overwhelming majority of the words of $A^{n}$ will lie in the interval

$$
\left[\left\lfloor\log _{d} n-1\right\rfloor, 2 \log _{d} n+\log _{d} \log _{d} n\right] .
$$

Proposition 3.2. Let $n>0$ and $r \geq 0$. The following holds:

$$
\operatorname{Card}\left(\left\{w \in A^{n} \mid K_{w} \geq\left\lceil\log _{d} n\right\rceil+r\right\}\right) \leq d^{n-r+1} .
$$

Proof. If $r=0$, the result is trivially true. Let us then suppose $r>0$ and set $m=\left\lceil\log _{d} n\right\rceil+r$. If $m>n$, then the set $\left\{w \in A^{n} \mid K_{w} \geq m\right\}$ is empty so that the statement follows. Let us then suppose $m \leq n$. By Proposition 2.4, one has

$$
\operatorname{Card}\left(\left\{w \in A^{n} \mid K_{w} \geq m\right\}\right) \leq(n-m+1) d^{n-m+1} \leq n d^{n-m+1} .
$$

Since $m=\left\lceil\log _{d} n\right\rceil+r$ one has $n d^{n-m+1} \leq d^{n-r+1}$, which proves the statement.

Now, we introduce the sequence $\phi_{n}$ of real numbers defined for any $n>1$ by

$$
\phi_{n}=\log _{d} n-2 \log _{d} \log _{d} n=\log _{d} \frac{n}{\log _{d}^{2} n} .
$$

Proposition 3.3. One has

$$
\lim _{n \rightarrow+\infty} \frac{1}{d^{n}} \operatorname{Card}\left(\left\{w \in A^{n} \mid K_{w} \leq \phi_{n}\right\}\right)=0 .
$$

Proof. First, we verify that for $1 \leq i \leq n$ one has

$$
\frac{1}{d^{n}} \operatorname{Card}\left(\left\{w \in A^{n} \mid K_{w} \leq i\right\}\right) \leq\left(1-\frac{1}{d^{i}}\right)^{\lfloor n / i\rfloor-1}
$$

Indeed, set $q=\lfloor n / i\rfloor$ and $r=n-i q$. Any word $w \in A^{n}$ such that $K_{w} \leq i$ can be factorized as

$$
w=s^{\prime} s_{1} s_{2} \cdots s_{q}
$$


with $s_{q} \in A^{i}, s_{1}, \ldots, s_{q-1} \in A^{i} \backslash\left\{s_{q}\right\}, s^{\prime} \in A^{r}$, since $s_{q}$ is unrepeated in $w$. The number of words of this kind is $d^{r} d^{i}\left(d^{i}-1\right)^{q-1}$, so that

$$
\operatorname{Card}\left(\left\{w \in A^{n} \mid K_{w} \leq i\right\}\right) \leq d^{r} d^{i}\left(d^{i}-1\right)^{q-1} .
$$

Dividing by $d^{n}$, one obtains equation (20). Using the inequality $1+x \leq \mathrm{e}^{x}$ holding for all real number $x$, one derives

$$
\left(1-\frac{1}{d^{i}}\right)^{\lfloor n / i\rfloor-1} \leq \mathrm{e}^{-d^{-i}\lfloor n / i-1\rfloor} .
$$

Since both $\phi_{n}$ and $d^{-\phi_{n}} n / \phi_{n}$ are diverging sequences, if one replaces $i$ by $\left\lfloor\phi_{n}\right\rfloor$ in the right hand side of equation (21), one obtains a sequence which vanishes when $n$ diverges. Thus, the conclusion follows by taking $i=\left\lfloor\phi_{n}\right\rfloor$ in equation (20).

By taking $r=\left\lfloor\log _{d} \log _{d} n\right\rfloor$ in Proposition 3.2 and using Proposition 3.3, one derives that

$$
\lim _{n \rightarrow+\infty} \frac{1}{d^{n}} \operatorname{Card}\left(\left\{w \in A^{n} \mid \log _{d} n-2 \log _{d} \log _{d} n<K_{w}<\log _{d} n+\log _{d} \log _{d} n\right\}\right)=1 .
$$

Thus, for a sufficiently large $n$, the minimal length of an unrepeated suffix in the overwhelming majority of the words of $A^{n}$ will be in the interval

$$
\left[\log _{d} n-2 \log _{d} \log _{d} n, \log _{d} n+\log _{d} \log _{d} n\right] .
$$

Now consider the map $D_{R}^{*}$ defined for all $i, n \geq 0$ (see Sect. 5 of CP) by

$$
D_{R}^{*}(i, n)=\operatorname{Card}\left(\left\{w \in A^{n} \mid R_{w} \geq i\right\}\right)=\sum_{m \geq i} D_{R}(m, n) .
$$

Since for any $w \in A^{n}$ one has $R_{w} \leq G_{w}+1$, one derives that for $0<m \leq n$

$$
D_{R}^{*}(m, n) \leq D_{G}^{*}(m-1, n) .
$$

Consequently, for a sufficiently large $n$ the maximal length of right special factors in the overwhelming majority of the words of $A^{n}$ will not exceed $\left\lceil 2 \log _{d} n+\right.$ $\left.\log _{d} \log _{d} n\right\rceil$.

Proposition 3.4. Let $n>0$ and $r>0$. The following holds:

$$
\operatorname{Card}\left(\left\{w \in A^{n} \mid R_{w} \leq\left\lfloor\log _{d} n\right\rfloor-r\right\}\right) \leq d^{n-r+2} .
$$

Proof. By Lemma 1.2, if $R_{w} \leq\left\lfloor\log _{d} n\right\rfloor-r$, then

$$
K_{w} \geq 2\left\lfloor\log _{d} n\right\rfloor-R_{w} \geq\left\lfloor\log _{d} n\right\rfloor+r \geq\left\lceil\log _{d} n\right\rceil+r-1 .
$$

Hence, by Proposition 3.2, the result follows. 
By taking $r=\left\lfloor\log _{d} \log _{d} n\right\rfloor$ in Propositions 3.4 and 3.1 and using equation (22), one derives that

$\lim _{n \rightarrow+\infty} \frac{1}{d^{n}} \operatorname{Card}\left(\left\{w \in A^{n} \mid \log _{d} n-\log _{d} \log _{d} n<R_{w}<2 \log _{d} n+\log _{d} \log _{d} n\right\}\right)=1$.

In other terms, for a sufficiently large $n$ the maximal length of right special factors in the overwhelming majority of the words of $A^{n}$ lies in the interval

$$
\left[\log _{d} n-\log _{d} \log _{d} n, 2 \log _{d} n+\log _{d} \log _{d} n\right] .
$$

Now, for any $n>0$, let us denote by $\langle G\rangle_{n},\langle R\rangle_{n}$, and $\langle K\rangle_{n}$, the average values of the parameters $G_{w}, R_{w}$, and $K_{w}$ on the words of length $n$, i.e.

$$
\langle G\rangle_{n}=\frac{1}{d^{n}} \sum_{w \in A^{n}} G_{w}, \quad\langle R\rangle_{n}=\frac{1}{d^{n}} \sum_{w \in A^{n}} R_{w}, \quad\langle K\rangle_{n}=\frac{1}{d^{n}} \sum_{w \in A^{n}} K_{w}
$$

Note that, for any $n>0$, one has

$$
\langle G\rangle_{n}=\frac{1}{d^{n}} \sum_{i=0}^{n} i D_{G}(i, n)=\frac{1}{d^{n}} \sum_{i=1}^{n} D_{G}^{*}(i, n) .
$$

In a similar way, one has

$$
\langle R\rangle_{n}=\frac{1}{d^{n}} \sum_{i=0}^{n} i D_{R}(i, n)=\frac{1}{d^{n}} \sum_{i=1}^{n} D_{R}^{*}(i, n)
$$

and

$$
\langle K\rangle_{n}=\frac{1}{d^{n}} \sum_{i=0}^{n} i D_{K}(i, n)=\frac{1}{d^{n}} \sum_{i=1}^{n} D_{K}^{*}(i, n) .
$$

In the case $d=2$, the values of $\langle R\rangle_{n},\langle K\rangle_{n}$, and $\langle G\rangle_{n}$ for $1 \leq n \leq 26$ are given in Table 1.

We recall that, as proved in Sections 2 and 3 of $\mathrm{CP}$, for all $w \in A^{+}$the following relations hold:

$$
K_{w} \leq K_{x w} \leq 1+K_{w}, \quad R_{w} \leq R_{x w} \leq 1+R_{w}, \quad G_{w} \leq G_{x w} \leq 1+G_{w} .
$$

Proposition 3.5. For any $n>0$ one has:

$$
\begin{aligned}
\langle K\rangle_{n} & <\langle K\rangle_{n+1}<1+\langle K\rangle_{n} \\
\langle R\rangle_{n} & <\langle R\rangle_{n+1}<1+\langle R\rangle_{n}, \\
\langle G\rangle_{n} & <\langle G\rangle_{n+1}<1+\langle G\rangle_{n} .
\end{aligned}
$$


Proof. By equation (26), for any $w \in A^{+}$and any $x \in A$ one has $K_{w} \leq K_{x w} \leq$ $1+K_{w}$. Moreover, for any $n>0$ there exist certainly a word $w \in A^{n}$ and letters $x, y \in A$ such that $K_{w}<K_{x w}$ and $K_{y w}<1+K_{w}$. For instance, one can take $w=a^{n}, x=a$, and $y=b \neq a$. Since

$$
d^{n+1}\langle K\rangle_{n+1}=\sum_{u \in A^{n+1}} K_{u}=\sum_{w \in A^{n}} \sum_{x \in A} K_{x w}
$$

one derives

$$
d \sum_{w \in A^{n}} K_{w}<d^{n+1}\langle K\rangle_{n+1}<d \sum_{w \in A^{n}}\left(1+K_{w}\right) .
$$

Dividing by $d^{n+1}$ one derives $\langle K\rangle_{n}<\langle K\rangle_{n+1}<1+\langle K\rangle_{n}$.

TABLE $1 .\langle R\rangle_{n},\langle K\rangle_{n}$, and $\langle G\rangle_{n}, 1 \leq n \leq 26$.

\begin{tabular}{r|rrr}
$n$ & $\langle R\rangle_{n}$ & $\langle K\rangle_{n}$ & $\langle G\rangle_{n}$ \\
\hline 1 & 0.0000 & 1.0000 & 0.0000 \\
2 & 0.5000 & 1.5000 & 0.5000 \\
3 & 1.0000 & 2.0000 & 1.2500 \\
4 & 1.6250 & 2.3750 & 1.6250 \\
5 & 2.1250 & 2.7500 & 2.1875 \\
6 & 2.6563 & 3.0625 & 2.5938 \\
7 & 3.1250 & 3.3438 & 2.9688 \\
8 & 3.5469 & 3.5859 & 3.2969 \\
9 & 3.9219 & 3.8125 & 3.6523 \\
10 & 4.2871 & 4.0117 & 3.9648 \\
11 & 4.6260 & 4.1895 & 4.2422 \\
12 & 4.9341 & 4.3516 & 4.4980 \\
13 & 5.2161 & 4.5005 & 4.7446 \\
14 & 5.4803 & 4.6372 & 4.9758 \\
15 & 5.7281 & 4.7633 & 5.1934 \\
16 & 5.9607 & 4.8800 & 5.3961 \\
17 & 6.1784 & 4.9886 & 5.5889 \\
18 & 6.3836 & 5.0903 & 5.7730 \\
19 & 6.5783 & 5.1856 & 5.9480 \\
20 & 6.7632 & 5.2754 & 6.1146 \\
21 & 6.9389 & 5.3602 & 6.2736 \\
22 & 7.1062 & 5.4405 & 6.4255 \\
23 & 7.2658 & 5.5167 & 6.5705 \\
24 & 7.4182 & 5.5893 & 6.7094 \\
25 & 7.5638 & 5.6586 & 6.8427 \\
26 & 7.7033 & 5.7249 & 6.9709
\end{tabular}


By equation (26), for any $w \in A^{+}$and any $x \in A$ one has $R_{w} \leq R_{x w} \leq 1+R_{w}$ and $G_{w} \leq G_{x w} \leq 1+G_{w}$. Moreover, for any $n$ there exist certainly a word $w \in A^{n}$ and letters $x, y \in A$ such that $R_{w}<R_{y w}, R_{x w}<1+R_{w}, G_{w}<G_{x w}$ and $G_{y w}<1+G_{w}$. For instance, one can take $w=a^{n}, x=a$, and $y=b \neq a$. Thus, similarly to the case of $K_{w}$, one derives $\langle R\rangle_{n}<\langle R\rangle_{n+1}<1+\langle R\rangle_{n}$ and $\langle G\rangle_{n}<\langle G\rangle_{n+1}<1+\langle G\rangle_{n}$.

Proposition 3.6. For any $n>1$,

$$
\langle G\rangle_{n} \leq\left\lceil 2 \log _{d} n\right\rceil-\frac{1}{2}
$$

Proof. By Lemma 1.3 and Proposition 2.9 one derives, for any $q>0$,

$$
\sum_{w \in A^{n}} G_{w} \leq \sum_{w \in A^{n}}\left(P_{w}(q)+q-1\right)=\left(\begin{array}{c}
n-q+1 \\
2
\end{array}\right) d^{n-q}+d^{n}(q-1) .
$$

Hence,

$$
\langle G\rangle_{n} \leq\left(\begin{array}{c}
n-q+1 \\
2
\end{array}\right) d^{-q}+q-1
$$

For $q=\left\lceil 2 \log _{d} n\right\rceil$ one has

$$
\left(\begin{array}{c}
n-q+1 \\
2
\end{array}\right) d^{-q}+q-1 \leq \frac{1}{2}+\left\lceil 2 \log _{d} n\right\rceil-1
$$

which concludes the proof.

Remark 3.7. In the case $d=2$ by taking $q=\left\lceil 2 \log _{2} n\right\rceil-1$ in equation (27) one gets for any $n>1,\langle G\rangle_{n} \leq\left\lceil 2 \log _{2} n\right\rceil-1$.

We observe that by equation (11) one has trivially for all $n>0$

$$
\langle G\rangle_{n} \geq\left\lfloor\log _{d} n\right\rfloor-1 .
$$

Proposition 3.8. For any $n>0$,

$$
\langle K\rangle_{n} \leq\left\lceil\log _{d} n\right\rceil+2
$$

Proof. By equation (25), one has

$$
\langle K\rangle_{n}=\frac{1}{d^{n}} \sum_{m=1}^{n} D_{K}^{*}(m, n) .
$$

We set $t=\left\lceil\log _{d} n\right\rceil+1$. In the previous sum, we majorize $D_{K}^{*}(m, n)$ by $d^{n}$ for $1 \leq m<t$ and by $d^{n-m+t}$ for $t \leq m \leq n$, in view of Proposition 3.2. One has 
then

$$
\langle K\rangle_{n} \leq \frac{1}{d^{n}}\left(\sum_{m=1}^{t-1} d^{n}+\sum_{m=t}^{n} d^{n-m+t}\right) \leq t-1+\frac{d}{d-1} \leq t+1 .
$$

Thus $\langle K\rangle_{n} \leq\left\lceil\log _{d} n\right\rceil+2$.

Proposition 3.9. One has

$$
\lim _{n \rightarrow+\infty} \frac{\langle K\rangle_{n}}{\log _{d} n}=1 .
$$

Proof. One has

$$
d^{n}\langle K\rangle_{n}=\sum_{w \in A^{n}} K_{w} \geq \operatorname{Card}\left(\left\{w \in A^{n} \mid K_{w}>\phi_{n}\right\}\right) \phi_{n},
$$

where $\phi_{n}$ is the sequence defined by equation (19). Thus in view of Proposition 3.8 one has

$$
\frac{1}{d^{n}} \operatorname{Card}\left(\left\{w \in A^{n} \mid K_{w}>\phi_{n}\right\}\right) \frac{\phi_{n}}{\log _{d} n} \leq \frac{\langle K\rangle_{n}}{\log _{d} n} \leq \frac{\left\lceil\log _{d} n\right\rceil+2}{\log _{d} n} .
$$

By Proposition 3.3 one derives

$$
\lim _{n \rightarrow+\infty} \frac{1}{d^{n}} \operatorname{Card}\left(\left\{w \in A^{n} \mid K_{w}>\phi_{n}\right\}\right)=1 .
$$

Moreover, $\lim _{n \rightarrow+\infty} \phi_{n} / \log _{d} n=\lim _{n \rightarrow+\infty}\left(\left\lceil\log _{d} n\right\rceil+2\right) / \log _{d} n=1$, so that the conclusion follows from equation (29).

Proposition 3.10. For $n>1$, one has

$$
d\langle R\rangle_{n}=\langle R\rangle_{n-1}+(d-1)\left(\langle G\rangle_{n-1}+1\right) .
$$

Proof. By equations (24) and (2) one has

$$
\begin{aligned}
d^{n}\langle R\rangle_{n} & =\sum_{i=1}^{n} i D_{R}(i, n) \\
& =\sum_{i=1}^{n} i D_{R}(i, n-1)+(d-1) \sum_{i=1}^{n} i D_{G}(i-1, n-1) \\
& =d^{n-1}\langle R\rangle_{n-1}+(d-1)\left(d^{n-1}\langle G\rangle_{n-1}+d^{n-1}\right) .
\end{aligned}
$$

Dividing by $d^{n-1}$, the statement follows. 
Corollary 3.11. For $n>0$, one has

$$
\langle R\rangle_{n}=(d-1) \sum_{m=1}^{n-1} \frac{\langle G\rangle_{m}+1}{d^{n-m}} .
$$

Proof. By Proposition 3.10 one has

$$
\langle R\rangle_{n}=\frac{1}{d}\langle R\rangle_{n-1}+\frac{d-1}{d}\left(\langle G\rangle_{n-1}+1\right) .
$$

By iteration, since $\langle R\rangle_{1}=0$, the result follows.

Corollary 3.12. For all $n>1$, one has

$$
\langle G\rangle_{n-1}+\frac{d-2}{d-1}<\langle R\rangle_{n}<\langle G\rangle_{n-1}+1 .
$$

Proof. By Proposition 3.5 one has $\langle R\rangle_{n-1}<\langle R\rangle_{n}$. Thus, from Proposition 3.10 it follows

$$
d\langle R\rangle_{n}<\langle R\rangle_{n}+(d-1)\left(\langle G\rangle_{n-1}+1\right)
$$

from which one has $\langle R\rangle_{n}\left\langle\langle G\rangle_{n-1}+1\right.$.

From Propositions 3.10 and 3.5 one gets

$$
d\langle R\rangle_{n}=\langle R\rangle_{n-1}+(d-1)\left(\langle G\rangle_{n-1}+1\right)>\langle R\rangle_{n}-1+(d-1)\left(\langle G\rangle_{n-1}+1\right) .
$$

Thus

$$
\langle R\rangle_{n}>\langle G\rangle_{n-1}+1-\frac{1}{d-1},
$$

from which the assertion follows.

Corollary 3.13. For all $n>1$, one has

$$
\langle R\rangle_{n} \geq\left\lfloor\log _{d}(n-1)\right\rfloor .
$$

Proof. By Proposition 3.10 one has

$$
d\langle R\rangle_{n}=\langle R\rangle_{n-1}+\langle G\rangle_{n-1}+1+(d-2)\left(\langle G\rangle_{n-1}+1\right) .
$$

By Lemma 1.1 one has

$$
\langle R\rangle_{n-1}+\langle G\rangle_{n-1}+1 \geq 2\left\lfloor\log _{d}(n-1)\right\rfloor
$$

and by equation (28) one obtains

$$
\langle G\rangle_{n-1}+1 \geq\left\lfloor\log _{d}(n-1)\right\rfloor,
$$


so that by equation (30) one derives

$$
d\langle R\rangle_{n} \geq d\left\lfloor\log _{d}(n-1)\right\rfloor,
$$

which concludes the proof.

For all $i, n \geq 0$ we set

$$
D_{K}^{>}(i, n)=\operatorname{Card}\left(\left\{w \in A^{n} \mid K_{w}=i>R_{w}\right\}\right)
$$

In Section 4 of CP we proved the following relation between $D_{G}^{*}$ and $D_{K}^{>}$: for $i \geq 0$ and $n>1$,

$$
D_{G}^{*}(i, n)=d D_{G}^{*}(i, n-1)+D_{K}^{>}(i+1, n) .
$$

From it the following noteworthy proposition follows.

Proposition 3.14. For $n>1$, one has

$$
\langle G\rangle_{n}=\langle G\rangle_{n-1}+\frac{1}{d^{n}} \operatorname{Card}\left(\left\{w \in A^{n} \mid K_{w}>R_{w}\right\}\right) .
$$

Proof. By equation (32) one has

$$
\sum_{i=0}^{n} D_{G}^{*}(i, n)=d \sum_{i=0}^{n} D_{G}^{*}(i, n-1)+\sum_{i=0}^{n} D_{K}^{>}(i+1, n) .
$$

Since, by equation (23),

$$
\sum_{i=0}^{n} D_{G}^{*}(i, n)=d^{n}\left(1+\langle G\rangle_{n}\right)
$$

and

$$
\sum_{i=0}^{n} D_{K}^{>}(i+1, n)=\operatorname{Card}\left(\left\{w \in A^{n} \mid K_{w}>R_{w}\right\}\right),
$$

one derives

$$
d^{n}\langle G\rangle_{n}+d^{n}=d^{n}\langle G\rangle_{n-1}+d^{n}+\operatorname{Card}\left(\left\{w \in A^{n} \mid K_{w}>R_{w}\right\}\right),
$$

which proves the assertion.

Lemma 3.15. Let $\mathcal{C}$ be a conjugacy class of $A^{+}$. Then one has

$$
\operatorname{Card}\left(\left\{w \in \mathcal{C} \mid K_{w}>R_{w}\right\}\right) \leq 2\left(\max _{w \in \mathcal{C}} K_{w}-1\right)
$$


Proof. Set $t=\max _{w \in \mathcal{C}} K_{w}$ and let $v \in \mathcal{C}$ be such that $K_{v}=t$. Let us verify that for any $u \in \mathcal{C}$, if $k_{v}^{\prime}$ has two non-terminal occurrences in $u$, then $R_{u} \geq K_{u}$. Indeed, either $k_{v}^{\prime}$ is a right special factor of $u$ or it can be extended on the right in a repeated factor $k_{v}^{\prime} x, x \in A$, of $u$ of length $t$. In the first case,

$$
R_{u} \geq t \geq K_{u}
$$

in the second case,

$$
G_{u} \geq t \geq K_{u},
$$

so that, in view of equation (1), $G_{u}=R_{u}-1$ and $K_{u}<R_{u}$.

To complete the proof, it is sufficient to observe that $\mathcal{C}$ contains at most $2(t-1)$ words which do not have two non-terminal occurrences of $k_{v}^{\prime}$.

Proposition 3.16. For any $n>1$ one has

$$
\frac{1}{d(n-1)} \leq \frac{1}{d^{n}} \operatorname{Card}\left(\left\{w \in A^{n} \mid K_{w}>R_{w}\right\}\right)<\frac{6 \log _{d} n+1}{n} .
$$

Proof. Let $t$ be a fixed integer such that $1 \leq t \leq n / 2$. Let $S$ be the set of the conjugacy classes $\mathcal{C} \subseteq A^{n}$ such that $\max _{v \in \mathcal{C}} K_{v} \leq t$ and $T$ be the set of the remaining conjugacy classes $\mathcal{C} \subseteq A^{n}$. One has that

$$
\begin{aligned}
& \operatorname{Card}\left(\left\{w \in A^{n} \mid K_{w}>R_{w}\right\}\right) \leq \\
& \quad \sum_{\mathcal{C} \in S} \operatorname{Card}\left(\left\{w \in \mathcal{C} \mid K_{w}>R_{w}\right\}\right)+\sum_{\mathcal{C} \in T} \operatorname{Card}\left(\left\{w \in \mathcal{C} \mid K_{w}>R_{w}\right\}\right) .
\end{aligned}
$$

A conjugacy class $\mathcal{C} \in S$ is primitive since for a non-primitive word $w, K_{w}>n / 2$. Since the number of primitive words of length $n$ is $\psi(n)$, there are $\psi(n) / n$ primitive conjugacy classes included in $A^{n}$ and, therefore,

$$
\operatorname{Card}(S) \leq \frac{\psi(n)}{n}<\frac{d^{n}}{n} .
$$

By Lemma 3.15, for any $\mathcal{C} \in S$, one has

$$
\operatorname{Card}\left(\left\{w \in \mathcal{C} \mid K_{w}>R_{w}\right\}\right) \leq 2(t-1),
$$

so that

$$
\sum_{\mathcal{C} \in S} \operatorname{Card}\left(\left\{w \in \mathcal{C} \mid K_{w}>R_{w}\right\}\right)<2 \frac{d^{n}}{n}(t-1) .
$$

Any conjugacy class $\mathcal{C} \in T$ contains at least one word $w$ such that $K_{w}>t$. By Proposition 2.4, the number of the words of length $n$ such that $K_{w}>t$ is 
upperbounded by $n d^{n-t}$. Thus, $\operatorname{Card}(T) \leq n d^{n-t}$. Since any conjugacy class of a word of length $n$ contains at most $n$ elements, one derives

$$
\sum_{\mathcal{C} \in T} \operatorname{Card}\left(\left\{w \in \mathcal{C} \mid K_{w}>R_{w}\right\}\right) \leq n^{2} d^{n-t} .
$$

Thus

$$
\operatorname{Card}\left(\left\{w \in A^{n} \mid K_{w}>R_{w}\right\}\right)<d^{n}\left(\frac{2(t-1)}{n}+\frac{n^{2}}{d^{t}}\right)
$$

Let us suppose that $n / 2 \geq\left\lceil 3 \log _{d} n\right\rceil$. Then, in the previous equation, we can take $t=\left\lceil 3 \log _{d} n\right\rceil$, obtaining

$$
\operatorname{Card}\left(\left\{w \in A^{n} \mid K_{w}>R_{w}\right\}\right)<d^{n} \frac{6 \log _{d} n+1}{n} .
$$

If, on the contrary, $n / 2<\left\lceil 3 \log _{d} n\right\rceil$, then $\left(6 \log _{d} n+1\right) / n \geq 1$, so that in any case one derives

$$
\frac{1}{d^{n}} \operatorname{Card}\left(\left\{w \in A^{n} \mid K_{w}>R_{w}\right\}\right)<\frac{6 \log _{d} n+1}{n} .
$$

Now, let us verify that

$$
\frac{1}{d(n-1)}<\frac{1}{d^{n}} \operatorname{Card}\left(\left\{w \in A^{n} \mid K_{w}>R_{w}\right\}\right) .
$$

We remark that in any conjugacy class $\mathcal{C}$ there is at least one word $w$ such that $K_{w} \geq R_{w}$. Indeed, let $t=\max _{v \in \mathcal{C}} G_{v}$. Then there is at least one word $w \in \mathcal{C}$ having a repeated suffix of length $t$. For such a $w, K_{w}=1+t=1+G_{w} \geq R_{w}$. For any $n>1$, the number of conjugacy classes included in $A^{n-1}$ is greater than or equal to $d^{n-1} /(n-1)$. Hence,

$$
\operatorname{Card}\left(\left\{w \in A^{n-1} \mid K_{w} \geq R_{w}\right\}\right) \geq \frac{d^{n-1}}{n-1} .
$$

By equation (8) for any word $w \in A^{n-1}$ such that $K_{w} \geq R_{w}$ there exists at least one letter $x \in B_{w}$ such that $K_{w x}=K_{w}+1$ and $R_{w x}=R_{w}$, so that $K_{w x}>R_{w x}$. Then one derives that

$$
\operatorname{Card}\left(\left\{v \in A^{n} \mid K_{v}>R_{v}\right\}\right) \geq \operatorname{Card}\left(\left\{w \in A^{n-1} \mid K_{w} \geq R_{w}\right\}\right) \geq \frac{d^{n-1}}{n-1} .
$$

From this, the result follows.

Remark 3.17. The class of words $w \in A^{*}$ such that $R_{w}<K_{w}$ has been introduced in [2]. We recall that these words can be also characterized as the words $w \in A^{*}$ which can be prolonged on the right in ultimately periodic words without 
adding new factors of length $1+R_{w}$. This class properly contains the class of semiperiodic words, i.e., the words $w$ such that $R_{w}<H_{w}$ [2]. In fact, as proved in [2], for a semiperiodic word $w, R_{w}=L_{w}<H_{w}=K_{w}$. As a consequence of Proposition 3.16, one has that the fraction of the words of length $n$ which are semiperiodic is upperbounded by $\left(6 \log _{d} n+1\right) / n$.

The following corollary shows that $\lim _{n \rightarrow \infty}\left(\langle G\rangle_{n}-\langle G\rangle_{n-1}\right)=0$.

Corollary 3.18. For $n>1$, one has

$$
\frac{1}{d(n-1)} \leq\langle G\rangle_{n}-\langle G\rangle_{n-1}<\frac{6 \log _{d} n+1}{n} .
$$

Proof. The result follows from the preceding proposition and Proposition 3.14.

Lemma 3.19. For any $n>0$ set $V=\left\{v \in A^{n} \mid K_{v}>R_{v}\right\}$. One has

$$
\sum_{w \in V} K_{w}<\frac{12 d^{n}}{n}\left(\log _{d} n+1\right)^{2} .
$$

Proof. Set $t=\left\lceil 2 \log _{d} n\right\rceil$. One has

$$
\begin{aligned}
\sum_{w \in V} K_{w} & =\sum_{i \geq 1} i \operatorname{Card}\left(\left\{w \in A^{n} \mid K_{w}=i \text { and } K_{w}>R_{w}\right\}\right) \\
& =\sum_{i \geq 1} \operatorname{Card}\left(\left\{w \in A^{n} \mid K_{w} \geq i \text { and } K_{w}>R_{w}\right\}\right) \\
& \leq \sum_{i=1}^{t} \operatorname{Card}\left(\left\{w \in A^{n} \mid K_{w}>R_{w}\right\}\right)+\sum_{i \geq t+1} \operatorname{Card}\left(\left\{w \in A^{n} \mid K_{w} \geq i\right\}\right) .
\end{aligned}
$$

By Proposition 3.16, as $t<2 \log _{d} n+1$, one has

$$
\sum_{i=1}^{t} \operatorname{Card}\left(\left\{w \in A^{n} \mid K_{w}>R_{w}\right\}\right)<t d^{n} \frac{6 \log _{d} n+1}{n}<\frac{d^{n}}{n}\left(12 \log _{d}^{2} n+8 \log _{d} n+1\right) .
$$

By Proposition 2.4 one has

$$
\sum_{i \geq t+1} \operatorname{Card}\left(\left\{w \in A^{n} \mid K_{w} \geq i\right\}\right) \leq \sum_{i \geq t+1} n d^{n-i+1}=n d^{n-t} \sum_{m=0}^{\infty} d^{-m} \leq 2 \frac{d^{n}}{n} .
$$

Thus,

$$
\sum_{w \in V} K_{w}<\frac{d^{n}}{n}\left(12 \log _{d}^{2} n+8 \log _{d} n+3\right) \leq \frac{12 d^{n}}{n}\left(\log _{d} n+1\right)^{2},
$$

which concludes the proof. 
The following proposition shows that for a sufficiently large $n,\langle R\rangle_{n}$ is approximately given by $\langle G\rangle_{n}+1$ :

Proposition 3.20. One has $\lim _{n \rightarrow \infty}\left(\langle R\rangle_{n}-\langle G\rangle_{n}\right)=1$.

Proof. For any $n>0$ one has:

$$
\langle G\rangle_{n}+1-\langle R\rangle_{n}=\frac{1}{d^{n}} \sum_{w \in A^{n}}\left(G_{w}+1-R_{w}\right) .
$$

By equation (1), if $K_{w} \leq R_{w}$, then $G_{w}+1-R_{w}=0$. If, on the contrary, $K_{w}>R_{w}$, then $G_{w}+1-R_{w}=K_{w}-R_{w} \leq K_{w}$. Hence,

$$
\langle G\rangle_{n}+1-\langle R\rangle_{n} \leq \frac{1}{d^{n}} \sum_{w \in V} K_{w}
$$

where $V=\left\{v \in A^{n} \mid K_{v}>R_{v}\right\}$. By Lemma 3.19, and since $\langle R\rangle_{n} \leq\langle G\rangle_{n}+1$, one derives

$$
0 \leq\langle G\rangle_{n}+1-\langle R\rangle_{n}<12 \frac{\left(\log _{d} n+1\right)^{2}}{n}
$$

From this, the statement follows.

Proposition 3.21. One has $\lim _{n \rightarrow \infty}\left(\langle R\rangle_{n}-\langle R\rangle_{n-1}\right)=0$.

Proof. By Proposition 3.10 one has

$$
\begin{aligned}
\langle R\rangle_{n}-\langle R\rangle_{n-1} & =(d-1)\left(\langle G\rangle_{n-1}+1-\langle R\rangle_{n}\right) \\
& =(d-1)\left(\langle G\rangle_{n-1}-\langle G\rangle_{n}+\langle G\rangle_{n}+1-\langle R\rangle_{n}\right) .
\end{aligned}
$$

By the previous proposition, $\lim _{n \rightarrow \infty}\left(\langle G\rangle_{n}+1-\langle R\rangle_{n}\right)=0$. By Corollary 3.18, $\lim _{n \rightarrow \infty}\left(\langle G\rangle_{n-1}-\langle G\rangle_{n}\right)=0$, that implies $\lim _{n \rightarrow \infty}\left(\langle R\rangle_{n}-\langle R\rangle_{n-1}\right)=0$.

We recall that a symmetric word of order $m$ is any word $w$ such that $R_{w}=$ $K_{w}=m$. Let $S(m, n)$ denote the class of all symmetric words of length $n$ and order $m$ on the alphabet $A$. Let $D_{K}^{>}$be the map defined by equation (31). The following result was proved in Section 4 of $\mathrm{CP}$ : for $0 \leq i \leq n$ one has

$$
D_{K}^{>}(i, n)=\sum_{m=1}^{i} \sum_{w \in S(i-m, n-m)} \operatorname{Card}\left(B_{w}\right)
$$

Proposition 3.22. For any $n>0$ one has

$$
\operatorname{Card}\left(\left\{w \in A^{*}|| w \mid<n \text { and } R_{w}=K_{w}\right\}\right)<d^{n} \frac{6 \log _{d} n+1}{n}
$$


Proof. For $0 \leq i \leq n$ by equation (33) one has

$$
D_{K}^{>}(i, n)=\sum_{m=1}^{i} \sum_{w \in S(i-m, n-m)} \operatorname{Card}\left(B_{w}\right)
$$

so that, since for any word $w, \operatorname{Card}\left(B_{w}\right) \geq 1$,

$$
\sum_{i=1}^{n} D_{K}^{>}(i, n) \geq \sum_{i=1}^{n} \sum_{m=1}^{i} \operatorname{Card}(S(i-m, n-m)) \geq \operatorname{Card}\left(\bigcup_{i=1}^{n} \bigcup_{m=1}^{i} S(i-m, n-m)\right) .
$$

As one easily verifies, one has

$$
\bigcup_{i=1}^{n} \bigcup_{m=1}^{i} S(i-m, n-m)=\bigcup_{j=0}^{n-1} \bigcup_{p=j}^{n-1} S(j, p)=\left\{w \in A^{*}|| w \mid<n \text { and } R_{w}=K_{w}\right\}
$$

Hence,

$$
\begin{aligned}
\operatorname{Card}\left(\left\{w \in A^{*}|| w \mid<n \text { and } R_{w}=K_{w}\right\}\right) & \leq \sum_{i=1}^{n} D_{K}^{>}(i, n) \\
& =\operatorname{Card}\left(\left\{w \in A^{n} \mid K_{w}>R_{w}\right\}\right) .
\end{aligned}
$$

From Proposition 3.16 the conclusion follows.

As a consequence of the previous proposition, one derives that the fraction of the words of length $n$ which are symmetric is upperbounded by the quantity $6 d\left(\log _{d}(n+1)+1\right) /(n+1)$.

We recall that a word $w$ is called periodic-like [3] if $k_{w}^{\prime}$ (or $h_{w}^{\prime}$ ) has no internal occurrence in $w$. As proved in [3], the class of periodic words is properly included in the class of semiperiodic words and this latter is properly included in the class of periodic-like words. Let $P$ be the set of periodic-like words of $A^{*}$ and $D_{P}$ the map defined for all $i, n \geq 0$ by

$$
D_{P}(i, n)=\operatorname{Card}\left(\left\{w \in P \cap A^{n} \mid K_{w}=i\right\}\right) .
$$

In other terms, $D_{P}(i, n)$ gives the number of periodic-like words of length $n$ having the shortest unrepeated suffix of length $i$. In Section 4 of CP the following relation between the maps $D_{K}$ and $D_{P}$ was proved: for all $i, n>0$ one has

$$
D_{K}(i, n)=d D_{K}(i, n-1)+D_{P}(i, n)-D_{P}(i+1, n-1) .
$$

Proposition 3.23. For any $n>0$ one has

$$
\frac{\operatorname{Card}\left(P \cap A^{n}\right)}{d^{n}}=\langle K\rangle_{n}-\langle K\rangle_{n-1} .
$$


Proof. From equation (34) one derives

$$
\sum_{i=1}^{n} i D_{K}(i, n)=d \sum_{i=1}^{n} i D_{K}(i, n-1)+\sum_{i=1}^{n} i D_{P}(i, n)-\sum_{i=1}^{n} i D_{P}(i+1, n)
$$

Now,

$$
\sum_{i=1}^{n} i D_{P}(i, n)-\sum_{i=1}^{n} i D_{P}(i+1, n)=\sum_{i=1}^{n} D_{P}(i, n)=\operatorname{Card}\left(P \cap A^{n}\right) .
$$

Thus, by equation (25) one obtains

$$
d^{n}\langle K\rangle_{n}=d^{n}\langle K\rangle_{n-1}+\operatorname{Card}\left(P \cap A^{n}\right),
$$

which proves our assertion.

Proposition 3.24. One has

$$
\lim _{n \rightarrow+\infty} \frac{\operatorname{Card}\left(P \cap A^{n}\right)}{d^{n}}=0 .
$$

Proof. Let $\phi_{n}$ be the sequence defined by equation (19). By Proposition 3.3 one has that

$$
\frac{1}{d^{n}} \operatorname{Card}\left(\left\{w \in P \cap A^{n} \mid K_{w}<\phi_{n}\right\}\right)
$$

vanishes when $n$ diverges. We recall [3] that the minimal period of a periodic-like word $w \in A^{n}$ is given by $\pi_{w}=n-K_{w}+1$. Therefore, since the number of words of length $n$ having minimal period $p$ is not larger than $d^{p}$, one has

$$
\begin{aligned}
\frac{1}{d^{n}} \operatorname{Card}\left(\left\{w \in P \cap A^{n} \mid K_{w} \geq \phi_{n}\right\}\right) & \leq \frac{1}{d^{n}} \operatorname{Card}\left(\left\{w \in A^{n} \mid \pi_{w} \leq n-\phi_{n}+1\right\}\right) \\
& \leq \frac{1}{d^{n}} \sum_{p=1}^{n-\phi_{n}+1} d^{p}<d^{-\phi_{n}+2}
\end{aligned}
$$

Since $\phi_{n}$ diverges with $n$, one derives that $\operatorname{Card}\left(P \cap A^{n}\right) / d^{n}$ vanishes when $n$ diverges.

By Propositions 3.23 and 3.24 one obtains the following:

\section{Corollary 3.25.}

$$
\lim _{n \rightarrow+\infty}\left(\langle K\rangle_{n}-\langle K\rangle_{n-1}\right)=0 .
$$




\section{Points of MAXimum}

In this section we show that the points of maximum of $D_{G}(i, n)$, viewed as a function of $i$ with $n$ fixed but sufficiently large, lie between $\left\lfloor\log _{d} n\right\rfloor-1$ and $\left\lceil 2 \log _{d} n+\log _{d} \log _{d} n\right\rceil-1$. Similarly, the points of maximum of $D_{K}(i, n)$ and $D_{R}(i, n)$ lie, respectively, between 0 and $\left\lceil\log _{d} n+\log _{d} \log _{d} n\right\rceil+2$ and between $\left\lfloor\log _{d} n-\log _{d} \log _{d} n\right\rfloor-4$ and $\left\lceil 2 \log _{d} n+\log _{d} \log _{d} n\right\rceil$.

Proposition 4.1. There exists an integer $n_{0}$ such that for all $n \geq n_{0}$ one has

$$
\max _{0 \leq i \leq n} D_{G}(i, n)=\max \left\{D_{G}(i, n) \mid\left\lfloor\log _{d} n\right\rfloor-1 \leq i<\left\lceil 2 \log _{d} n+\log _{d} \log _{d} n\right\rceil\right\} .
$$

Proof. Let us take $n \geq d$ and set $m=\left\lceil 2 \log _{d} n+\log _{d} \log _{d} n\right\rceil$. Since $m>0$, from Corollary 2.8 one has

$$
\sum_{i \geq m} D_{G}(i, n)=\operatorname{Card}\left(\left\{w \in A^{n} \mid G_{w} \geq m\right\}\right)<\frac{d^{n}}{2 \log _{d} n} .
$$

Thus,

$$
\max _{i \geq m} D_{G}(i, n)<\frac{d^{n}}{2 \log _{d} n}
$$

By equation (35), one has

$$
\operatorname{Card}\left(\left\{w \in A^{n} \mid G_{w}<m\right\}\right)>\frac{d^{n}}{2 \log _{d} n}\left(2 \log _{d} n-1\right) .
$$

Thus, since by equation (11), $D_{G}(i, n)=0$ for $i<\left\lfloor\log _{d} n\right\rfloor-1$, one has

$$
\frac{d^{n}}{2 \log _{d} n}\left(2 \log _{d} n-1\right)<\sum_{i=\left\lfloor\log _{d} n\right\rfloor-1}^{m-1} D_{G}(i, n) \leq M\left(\log _{d} n+\log _{d} \log _{d} n+3\right)
$$

where $M=\max \left\{D_{G}(i, n) \mid\left\lfloor\log _{d} n\right\rfloor-1 \leq i<m\right\}$. Hence,

$$
M>\frac{d^{n}}{2 \log _{d} n} \frac{2 \log _{d} n-1}{\log _{d} n+\log _{d} \log _{d} n+3} .
$$

There exists an integer $n_{0} \geq d$ such that for all $n \geq n_{0}$ one has

$$
\frac{2 \log _{d} n-1}{\log _{d} n+\log _{d} \log _{d} n+3} \geq 1
$$


Thus, for $n \geq n_{0}$ one has

$$
M>\frac{d^{n}}{2 \log _{d} n},
$$

which, in view of equation (36), proves our assertion.

Proposition 4.2. There exists an integer $n_{0}$ such that for all $n \geq n_{0}$ one has

$$
\max _{0 \leq i \leq n} D_{K}(i, n)=\max \left\{D_{K}(i, n) \mid 0 \leq i<\left\lceil\log _{d} n+\log _{d} \log _{d} n+2\right\rceil\right\} .
$$

Proof. Let $n \geq d$ and set $m=\left\lceil\log _{d} n+\log _{d} \log _{d} n+2\right\rceil$. From Proposition 2.4 one has

$$
\sum_{i \geq m} D_{K}(i, n)<n d^{n-m+1} \leq \frac{d^{n-1}}{\log _{d} n}
$$

so that

$$
\max _{i \geq m} D_{K}(i, n)<\frac{d^{n-1}}{\log _{d} n}
$$

By equation (38), one has

$$
\operatorname{Card}\left(\left\{w \in A^{n} \mid K_{w}<m\right\}\right)>d^{n}\left(1-\frac{1}{d \log _{d} n}\right)=\frac{d^{n-1}}{\log _{d} n}\left(d \log _{d} n-1\right) .
$$

Thus,

$$
\frac{d^{n-1}}{\log _{d} n}\left(d \log _{d} n-1\right)<\sum_{i=1}^{m-1} D_{K}(i, n) \leq M\left(\log _{d} n+\log _{d} \log _{d} n+2\right)
$$

where $M=\max \left\{D_{K}(i, n) \mid 1 \leq i<m\right\}$. Hence,

$$
M>\frac{d^{n-1}}{\log _{d} n} \frac{d \log _{d} n-1}{\log _{d} n+\log _{d} \log _{d} n+2} .
$$

There exists an integer $n_{0} \geq d$ such that for all $n \geq n_{0}$ one has

$$
\frac{d \log _{d} n-1}{\log _{d} n+\log _{d} \log _{d} n+2} \geq 1 .
$$

Hence, for $n \geq n_{0}$ one has

$$
M>\frac{d^{n-1}}{\log _{d} n}
$$

which, in view of equation (39), proves our assertion. 
Proposition 4.3. There exists an integer $n_{0}$ such that for all $n \geq n_{0}$ one has

$$
\max _{0 \leq i \leq n} D_{R}(i, n)=\max _{m^{\prime}<i \leq m} D_{R}(i, n)
$$

where $m^{\prime}=\left\lfloor\log _{d} n-\log _{d} \log _{d} n-4\right\rfloor$ and $m=\left\lceil 2 \log _{d} n+\log _{d} \log _{d} n\right\rceil$.

Proof. Let us observe that

$$
\operatorname{Card}\left(\left\{w \in A^{n} \mid R_{w}>m\right\}\right) \leq \operatorname{Card}\left(\left\{w \in A^{n} \mid G_{w} \geq m\right\}\right)=D_{G}^{*}(m, n),
$$

so that, by equation (35),

$$
\operatorname{Card}\left(\left\{w \in A^{n} \mid R_{w}>m\right\}\right)<\frac{d^{n}}{2 \log _{d} n} .
$$

Since, as one easily derives, $m^{\prime} \leq\left\lfloor\log _{d} n\right\rfloor-\left\lceil 2+\log _{d}\left(2 \log _{d} n\right)\right\rceil$, by Proposition 3.4 one has

$$
\operatorname{Card}\left(\left\{w \in A^{n} \mid R_{w} \leq m^{\prime}\right\}\right) \leq \frac{d^{n}}{2 \log _{d} n}
$$

Consequently,

$$
\operatorname{Card}\left(\left\{w \in A^{n} \mid m^{\prime}<R_{w} \leq m\right\}\right)>d^{n}\left(1-\frac{1}{\log _{d} n}\right) .
$$

Thus, one derives that

$$
\max _{i>m} D_{R}(i, n) \leq \sum_{i>m} D_{R}(i, n)=\operatorname{Card}\left(\left\{w \in A^{n} \mid R_{w}>m\right\}\right)<\frac{d^{n}}{2 \log _{d} n}
$$

and

$$
\max _{i \leq m^{\prime}} D_{R}(i, n) \leq \sum_{i \leq m^{\prime}} D_{R}(i, n)=\operatorname{Card}\left(\left\{w \in A^{n} \mid R_{w} \leq m^{\prime}\right\}\right) \leq \frac{d^{n}}{2 \log _{d} n} .
$$

Moreover,

$$
\begin{aligned}
\max _{m^{\prime}<i \leq m} D_{R}(i, n) & \geq \frac{\sum_{i=m^{\prime}+1}^{m} D_{R}(i, n)}{m-m^{\prime}} \\
& =\frac{\operatorname{Card}\left(\left\{w \in A^{n} \mid m^{\prime}<R_{w} \leq m\right\}\right)}{m-m^{\prime}}>\frac{d^{n}}{\log _{d} n} \frac{\log _{d} n-1}{m-m^{\prime}} .
\end{aligned}
$$

As

$$
\lim _{n \rightarrow \infty} \frac{\log _{d} n-1}{m-m^{\prime}}=1
$$


one derives that for all sufficiently large $n$

$$
\max _{m^{\prime}<i \leq m} D_{R}(i, n)>\frac{d^{n}}{2 \log _{d} n},
$$

which proves our assertion.

Acknowledgements. The authors are thankful to Istituto di Cibernetica "E. Caianiello" del C.N.R. for its valuable support to this research.

\section{REFERENCES}

[1] A. Carpi and A. de Luca, Words and special factors. Theoret. Comput. Sci. 259 (2001) $145-182$.

[2] A. Carpi and A. de Luca, Semiperiodic words and root-conjugacy. Theoret. Comput. Sci. (to appear).

[3] A. Carpi and A. de Luca, Periodic-like words, periodicity, and boxes. Acta Informatica 37 (2001) 597-618.

[4] A. Carpi and A. de Luca, On the distribution of characteristic parameters of words. RAIRO: Theoret. Informatics Appl. 36 (2002) 99-128.

[5] A. Carpi, A. de Luca and S. Varricchio, Words, univalent factors, and boxes. Acta Informatica 38 (2002) 409-436.

[6] N.J. Fine and H.S. Wilf, Uniqueness theorem for periodic functions. Proc. Amer. Math. Soc. 16 (1965) 109-114.

[7] G.H. Hardy and E.M. Wright, An Introduction to the Theory of Numbers. Oxford University Press, Oxford, UK (1979).

[8] J.D. Kececioglu and E.W. Myers, Combinatorial algorithms for DNA sequence assembly. Algorithmica 13 (1995) 7-51.

[9] M. Lothaire, Combinatorics on Words, 2nd Edition. Cambridge Mathematical Library, Cambridge University Press, Cambridge, UK (1997).

[10] F. Mignosi, A. Restivo and M. Sciortino, Forbidden factors and fragment assembly. RAIRO: Theoret. Informatics Appl. (to appear).

Communicated by J. Berstel.

Received November 15, 2001. Accepted May 27, 2002.

To access this journal online: www.edpsciences.org 